\title{
A FDTD Simulation on YIG Based 2D Magnetic Photonic Crystal
}

\section{Liu Jianting, Wang Shuangbao*}

School of Optics and Electronic Information, Huazhong University of Science \& Technology, Wuhan, P.R. China

\section{Email address}

18771067614@163.com (Liu Jianting),Sb_wang@126.com (Wang Shuangbao)

${ }^{*}$ Corresponding author

\section{To cite this article:}

Liu Jianting, Wang Shuangbao. A FDTD Simulation on YIG Based 2D Magnetic Photonic Crystal. Science Discovery. Vol. 4, No. 4, 2016, pp. 253-259. doi: 10.11648/j.sd.20160404.19

Received: June 29, 2016; Accepted: June 30, 2016; Published: August 3, 2016

\begin{abstract}
This paper mentioned a FDTD simulation on the optical propagation in plane and along the $\mathrm{z}$ axis, we analysis the MPC structure, filling factor, dielectric constant, and their affects on the optical bandgap. This study put special attention on the polarization phenomena. Through simulation we observed the real time change of light (especially on polarization) in 2D MPC, obtaining the relationship curve between polarization angle and polarization degree to the MPC structure and filling factor. Based on FDTD aglorithm, this change reflects the Faraday effect from micro-level. Finally we suggest a dynamic measurement method based on Fetosecond laser, in order to realize time-divided Faraday effect.
\end{abstract}

Keywords: Magneto-Optical Effect, FDTD, Magnetic Photonic Crystal, Polarization Measurement

\section{YIG基二维磁性光子晶体的FDTD仿真研究}

\author{
刘鉴霆, 王双保* \\ 光学与电子信息学院, 华中科技大学, 武汉, 中国

\section{邮箱} \\ 18771067614@163.com（刘鉴霆）,Sb_wang@126.com（王双保）
}

\begin{abstract}
摘要: 本文基于FDTD算法, 运用了Matlab和Rsoft软件仿真了光在二维磁性光子晶体面内和轴向的传播过程。分析了磁 性光子晶体的晶格结构、填充率以及介电常数对光子带隙的影响。研究还从偏振出发, 观察了光在二维磁性光子晶体 沿纵向传播过程中偏振态的实时变化, 得到了偏转角和偏振度在磁性光子晶体结构与填充率等因素作用下的关系曲线。 基于FDTD算法本身的实时性, 这一变化曲线也从微观的角度上反映了磁光效应。最后提出一种基于飞秒激光器的磁光 偏振测量方法, 以控制光子数的方式来实现微观的磁光效应。
\end{abstract}

关键词: 磁光效应, FDTD, 磁性光子晶体, 偏振测量

\section{1. 引言}

光子晶体 (Photonic Crystal) 是现在科学研究的一 个热点问题, 它是一种由不同介电常数的介质周期性排列 而成的人工微结构, 它的晶格常数在波长量级, 能够改变
在其间传播的光的性质。由于介电常数存在空间上的周期 性, 所以它对光的折射率同样有周期性分部的影响, 在其 中传播的光波的色散曲线也会形成带状能带结构, 叫做光 子能带。光子能带之间可能出现带隙, 即光子带隙。频率 落在光子带隙内的光子, 在某些方向上是被严格禁止传播 
的。并且在光子晶体中引入缺陷, 能够使光局域在缺陷部 分，被称为光子局域效应 [1]。由于它在微波和光电子方 面具有广阔的应用前景，因此这种具有光子带隙和光子局 域特性的材料便成为人们广泛进行理论研究和实验研究 的主题。此次所研究的磁性光子晶体指的是由磁性材料构 成的光子晶体或引入磁性缺陷的光子晶体。磁性材料制作 成的PC由于其磁光效应强、Verdet常数大、体积小, 满足 系统集成化的要求而得到了广泛的关注。磁性材料可以通 过外加直流磁场 (或温度) 来调解自身的光学性质, 这使 得组分中含有磁性材料的光子晶体具有巨大的应用前景。 在磁性介质中传播的电磁波另一个重要特性是非倒易性; 倒易性是指当波矢 $\mathrm{k}$ 改变符号时光的偏振态也随之改变 (左旋偏振光变为右旋偏振光, 右旋变左旋)。

虽然目前在磁光偏振理论和仿真方面, 国内外已经有 一些单位做了这方面的科学研究, 然而他们大都还是把工 作建立在统计学的基础上, 而不是以动力学做基础 (后者 更适合研究高速光学现象), 再得出统计学的结论。所以, 在这里主要是采用了以时间微分为基础的电磁场动力学 算法, 既要记录偏振态变化的过程, 也要进行相关参数统 计、数据处理和状态概况。在仿真中进行模式数据迭代, 以节约运行内存资源。客观上, 磁性光子晶体在时间微分 条件下的光学作用的机理有待深入研究, 在此领域存在着 许多迄今仍不是十分清楚的问题, 如: 光与磁性介质的作 用 (磁光作用) 的本质, 偏振态随角频率的变化, 非周期 性缺陷对偏振的影响等。到目前为止在时间微观层面很多 问题都还没有较好地诠释。一些磁性光子晶体基本面的问 题, 如非线性介电常数、磁导率的非对称角元在模拟仿真 中的作用也是近些年才集成到国际光学计算软件 (如 Rsoft和FDTD solution）中去的, 而对偏振动力学相关的 研究, 人们在对其认识上实际还有很多的盲点。这也正是 本课题组一直在努力要解决的问题。

\section{YIG基磁性光子晶体的偏振态仿真}

偏振态是光束的重要特征参数之一, 对于偏振态的表 征方式有两种, 一种是琼斯矩阵法, 沿光传播方向上某点 以琼斯矩阵复振幅表示的偏振态定义为:

$$
\left[\begin{array}{c}
E_{x} \\
E_{y}
\end{array}\right]=e^{i(\omega t-k z)}\left[\begin{array}{l}
A e^{i \delta_{x}} \\
B e^{i \delta_{y}}
\end{array}\right]=e^{i\left(\omega t-k z+\delta_{x}\right)}\left[\begin{array}{c}
A \\
B e^{i \delta}
\end{array}\right]
$$

还有一种是斯托克斯参量表示法, 用四个参量可以表 示一切偏振态, 表示为 $\left(S_{0}, S_{1}, S_{2}, S_{3}\right)$, 光的电矢量 $\mathrm{S}$ 分量振 幅 $E_{s}$ 和 $\mathrm{P}$ 分量振幅 $E_{p}$ 及相位差 $\delta$ 与四个斯托克斯参量的关 系如下:

$$
\left.\begin{array}{c}
S_{0}=E_{p}^{2}+E_{s}^{2} \\
S_{1}=E_{p}^{2}-E_{s}^{2} \\
S_{2}=2 E_{p} E_{s} \cos \delta \\
S_{3}=2 E_{p} E_{s} \sin \delta
\end{array}\right\}
$$

斯托克斯参量法的四个分量是直接的光强分量, 因此 常用于高速实时测量偏振系统中 $[2,3]$ 。

\section{1. 定义}

本文基于FDTD算法的仿真中, 可以求出每个点上的Ex、 Ey分量, 因此是直接用Ex、Ey分量来表征其偏振态。

\subsection{FDTD 方法简介}

构造FDTD（时域有限差分）算法的出发点是麦克斯韦 时域方程:

$$
\begin{gathered}
\nabla \times H=\frac{\partial D}{\partial t}+J \\
\nabla \times E=-\frac{\partial B}{\partial t}-M \\
\nabla \cdot D=\rho_{e} \\
\nabla \cdot B=\rho_{m}
\end{gathered}
$$

式中: $E$ 为电场强度 $(\mathrm{V} / \mathrm{m}) ; \mathrm{D}$ 为电位移 $\left(\mathrm{C} / \mathrm{m}^{2}\right) ; \mathrm{H}$ 为磁 场强度 $(\mathrm{A} / \mathrm{m}) ； \mathrm{~B}$ 为磁通量密度 $\left(\mathrm{Wb} / \mathrm{m}^{2}\right) ； \mathrm{~J}$ 为电流密度 $\left(\mathrm{A} / \mathrm{m}^{2}\right) ; \mathrm{M}$ 为磁流密度 $\left(\mathrm{V} / \mathrm{m}^{2}\right) ; \rho_{e}$ 为电荷密度 $\left(\mathrm{C} / \mathrm{m}^{2}\right)$; $\rho_{m}$ 为磁荷密度 $\left(\mathrm{Wb} / \mathrm{m}^{2}\right)[4]$ 。

其中，对线性、各向同性和非色散煤质包含本构关系 有:

$$
\begin{aligned}
& D=\varepsilon \varepsilon_{0} E \\
& B=\mu \mu_{0} H
\end{aligned}
$$

将旋度方程差分化后有 [5]:

$$
\begin{aligned}
& \frac{\partial E_{x}}{\partial t}=\frac{1}{\varepsilon_{x}}\left(\frac{\partial H_{z}}{\partial y}-\frac{\partial H_{y}}{\partial z}-\sigma_{x}^{e} E_{x}-J_{i x}\right) \\
& \frac{\partial E_{y}}{\partial t}=\frac{1}{\varepsilon_{y}}\left(\frac{\partial H_{x}}{\partial z}-\frac{\partial H_{z}}{\partial x}-\sigma_{y}^{e} E_{y}-J_{i y}\right) \\
& \frac{\partial E_{x}}{\partial t}=\frac{1}{\varepsilon_{x}}\left(\frac{\partial H_{y}}{\partial x}-\frac{\partial H_{x}}{\partial y}-\sigma_{z}^{e} E_{z}-J_{i z}\right) \\
& \frac{\partial H_{x}}{\partial t}=\frac{1}{\mu_{x}}\left(\frac{\partial E_{y}}{\partial z}-\frac{\partial E_{z}}{\partial y}-\sigma_{x}^{m} H_{x}-M_{i x}\right) \\
& \frac{\partial H_{y}}{\partial t}=\frac{1}{\mu_{y}}\left(\frac{\partial E_{z}}{\partial x}-\frac{\partial E_{x}}{\partial z}-\sigma_{y}^{m} H_{y}-M_{i y}\right) \\
& \frac{\partial H_{x}}{\partial t}=\frac{1}{\mu_{x}}\left(\frac{\partial E_{x}}{\partial y}-\frac{\partial E_{y}}{\partial x}-\sigma_{z}^{m} H_{z}-M_{i z}\right)
\end{aligned}
$$




\section{3. 模型}

本文采用的是YIG基二维磁性光子晶体空气孔模型, 如图所示:

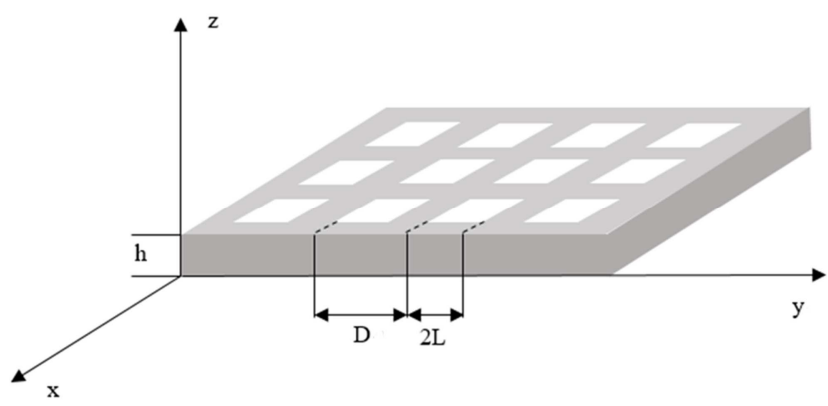

图1 二维磁性光子晶体方形空气孔结构。

在分析光子晶体时, 为简单起见, 一般将所用材料假 设为各向同性材料, 因此其介电张量可以简化为标量 $\varepsilon$ 。 根据相关文献对磁光材料介电张量的描述, 沿z方向磁化 的磁光材料的介电张量是一个复数张量, 可由下式表示:

$$
\mathcal{\varepsilon}=\left[\begin{array}{ccc}
\varepsilon_{0} & \varepsilon_{12} & 0 \\
\varepsilon_{21} & \varepsilon_{0} & 0 \\
0 & 0 & \varepsilon_{0}
\end{array}\right]
$$

式中张量元 $\varepsilon_{12}$ 和 $\varepsilon_{21}$ 为虚数, 且有 $\varepsilon_{12}=-\varepsilon_{21}=i \delta$ 。因此, 与分析传统的光子晶体方法不同, 需要对FDTD方法进行相 应的修正 $[6,7]$ 。

\subsection{1. 面内传播}

仿真二维磁性光子晶体的面内传播时, 仅对 TMz模进 行仿真, 由Ez表示传播过程中的光强变化。

将微分方程参量修改为:

$$
\begin{aligned}
& \frac{\partial E_{x, y, z}}{\partial t}=\frac{E_{x, y, z}^{n+1}-E_{x, y, z}^{n}}{\Delta t} \\
& \frac{\partial H_{x, y, z}}{\partial t}=\frac{H_{x, y, z}^{n+1}-H_{x, y, z}^{n}}{\Delta t} \\
& \frac{\partial E_{z}}{\partial x}=\frac{E_{z}^{n}(i, j)-E_{z}^{n}(i-1, j)}{\Delta x} \\
& E_{x}^{n+\frac{1}{2}}(i, j)=\frac{E_{x}^{n+1}(i, j)+E_{x}^{n}(i, j)}{2}
\end{aligned}
$$

因为面内传播的仿真中, 只能去得到 $E z$ 一个分量的变 化, 无法从中直接看出偏振态的变化, 所以只能去分析其 透过谱曲线, 来观察光子带隙等特性。

仿真中改变了光子晶体的晶格结构、填充率等参数, 最终得出的结论为: 对于二维空气柱型光子晶体而言, 不 同的晶格结构有着禁带结构, 一般而言, 晶格结构的对称 度越低, 禁带的宽度越大, 因此三角晶格结构比正方晶格 结构有着更大的禁带宽度。而对于同一种晶格结构而言,
其禁带结构还可以通过改变填充率和介电常数比来优化。 通常介电常数比越大, 光子晶体的禁带宽度越大。而对于 某种特定的光子晶体, 禁带宽度会随着填充率的增大而增 大, 并在某一范围达到最大值。下图为部分仿真图, 图3 和图4为两种环形器结构 [8]:

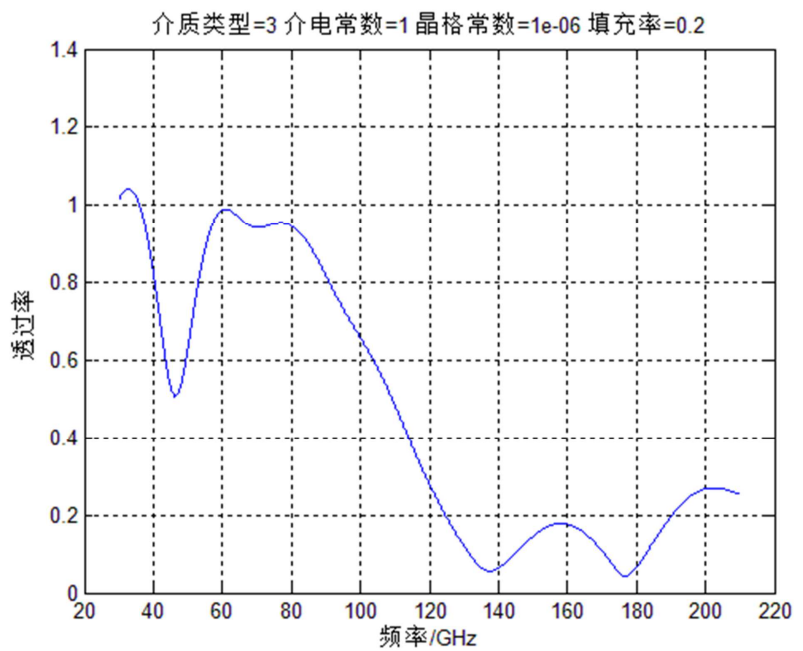

(a)

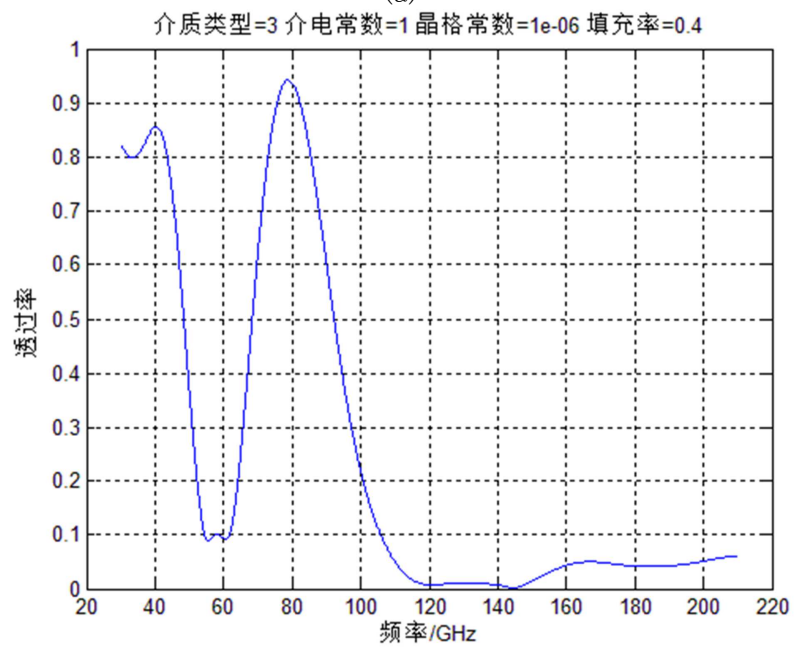

(b)

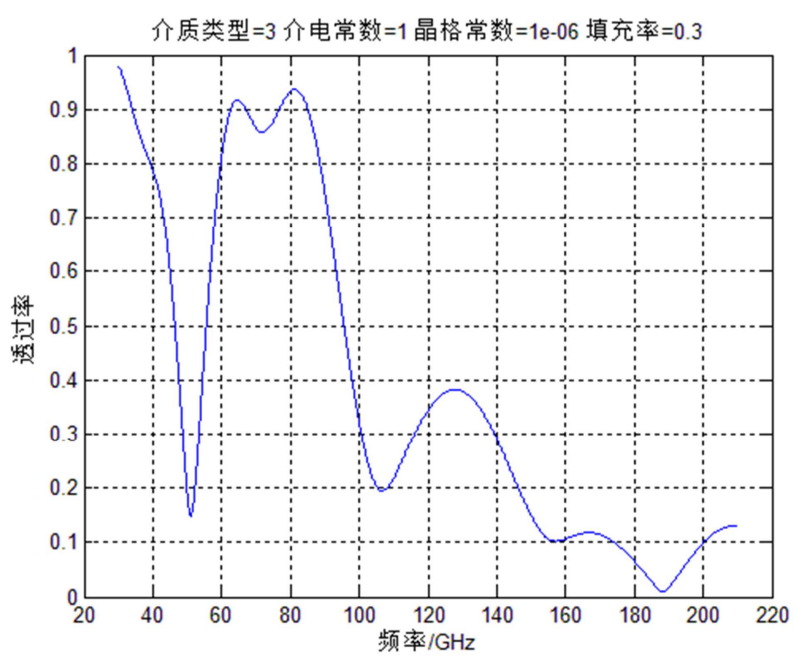

(c) 


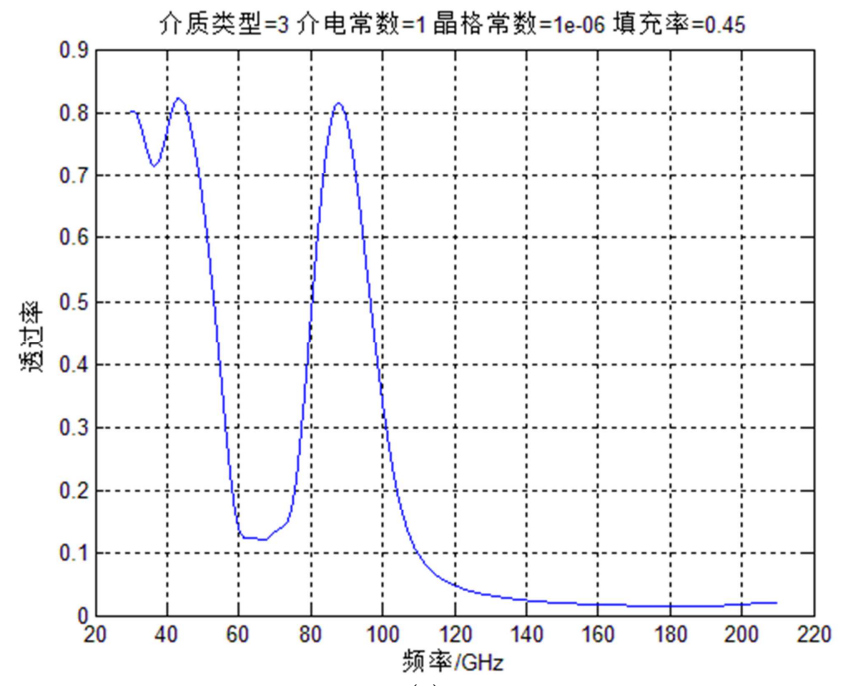

(d)

图2 填充率分别为 (a) 2 (b) 3 (c) 4 (d) 4.5 时的透过谱。

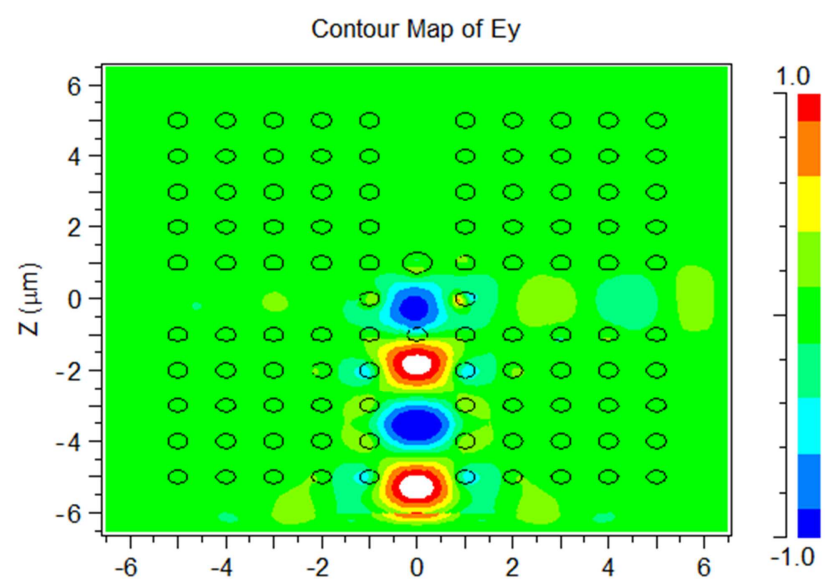

图3 二维磁性光子晶体结构 1 面内传播图。

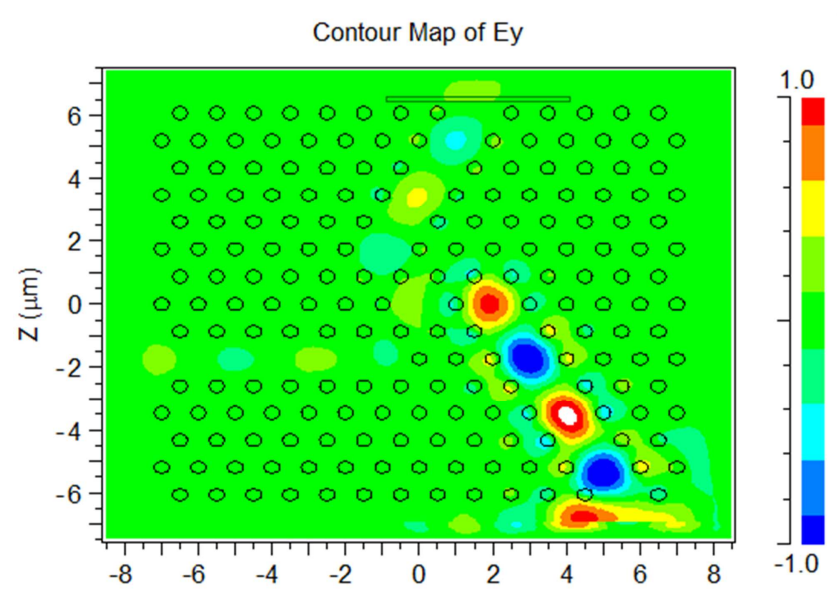

图4 二维磁性光子晶体结构 2 面内传播图。

\subsection{2. 沿纵向传播}

沿纵向传播时, 对二维平面内的一些假设依然成立, 但由于要计算6个分量, 因此推导会相对繁琐, 推导如下: 首先做一些定义, 令:

$$
\begin{aligned}
& M 1=\frac{2 \varepsilon-\Delta t \sigma_{x}(i, j)}{2 \varepsilon+\Delta t \sigma_{x}(i, j)} \\
& M 2=\frac{2 \Delta t}{2 \varepsilon+\Delta t \sigma_{x}(i, j)} \\
& N 1=\frac{2 \varepsilon-\Delta t \sigma_{y}(i, j)}{2 \varepsilon+\Delta t \sigma_{y}(i, j)} \\
& N 2=\frac{2 \Delta t}{2 \varepsilon+\Delta t \sigma_{y}(i, j)}
\end{aligned}
$$

代入到FDTD差分表达式中得到:

$$
\begin{aligned}
E_{x}{ }^{n+1} & (i, j, k)=A_{1}(i, j, k) \times E_{x}{ }^{n}(i, j, k) \\
+ & A_{2}(i, j, k) \times\left(H_{z}{ }^{n+1 / 2}(i, j+1, k)-H_{z}{ }^{n+1 / 2}(i, j, k)\right) \\
+ & A_{3}(i, j, k) \times\left(H_{y}{ }^{n+1 / 2}(i, j, k+1)-H_{y}{ }^{n+1 / 2}(i, j, k)\right) \\
+ & A_{4}(i, j, k) \times E_{y}{ }^{n}(i, j, k) \\
+ & A_{5}(i, j, k) \times\left(H_{x}{ }^{n+1 / 2}(i, j, k+1)-H_{x}{ }^{n+1 / 2}(i, j, k)\right) \\
+ & A_{6}(i, j, k) \times\left(H_{z}{ }^{n+1 / 2}(i+1, j, k)-H_{z}{ }^{n+1 / 2}(i, j, k)\right) \\
H_{x}^{n+1 / 2} & (i, j, k)=D_{1}(i, j, k) \times H_{x}^{n-1 / 2}(i, j, k) \\
& +D_{2}(i, j, k) \times\left(E_{y}^{n}(i, j, k+1)-E_{y}^{n}(i, j, k)\right) \\
& +D_{3}(i, j, k) \times\left(E_{z}^{n}(i, j+1, k)-E_{z}^{n}(i, j, k)\right)
\end{aligned}
$$

其中:

$$
\begin{aligned}
& A_{1}=\frac{\Delta t^{2} M_{1}-\delta^{2} M_{2} N_{2}}{\Delta t^{2}-\delta^{2} M_{2} N_{2}} \\
& A_{2}=\frac{\Delta t^{2} M_{2}}{\Delta y\left(\Delta t^{2}-\delta^{2} M_{2} N_{2}\right)} \\
& A_{3}=\frac{-\Delta t^{2} M_{2}}{\Delta z\left(\Delta t^{2}-\delta^{2} M_{2} N_{2}\right)} \\
& A_{4}=\frac{-i \delta \Delta t\left(N_{1}-1\right) M_{2}}{\Delta t^{2}-\delta^{2} M_{2} N_{2}} \\
& A_{5}=\frac{-i \delta \Delta t N_{2} M_{2}}{\Delta z\left(\Delta t^{2}-\delta^{2} M_{2} N_{2}\right)} \\
& A_{6}=\frac{i \delta \Delta t N_{2} M_{2}}{\Delta x\left(\Delta t^{2}-\delta^{2} M_{2} N_{2}\right)} \\
& D_{1}=\frac{2 \mu-\Delta t \sigma_{m}}{2 \mu+\Delta t \sigma_{m}} \\
& D_{2}=\frac{2 \Delta t}{\Delta z\left(2 \mu+\Delta t \sigma_{m}\right)} \\
& D_{3}=\frac{-2 \Delta t}{\Delta y\left(2 \mu+\Delta t \sigma_{m}\right)}
\end{aligned}
$$

其他分量同理推导。 
二维磁性光子晶体的条件为: 填充率 $\mathrm{f}=0.25$, 波长为 1. $3 \mathrm{um}$, 磁性角元分量 $\delta=1.98 \mathrm{e}-1$ 。输入光为 $\mathrm{x}$ 方向上振 幅为 0.5 的正弦线偏光。得到的部分仿真图如下:

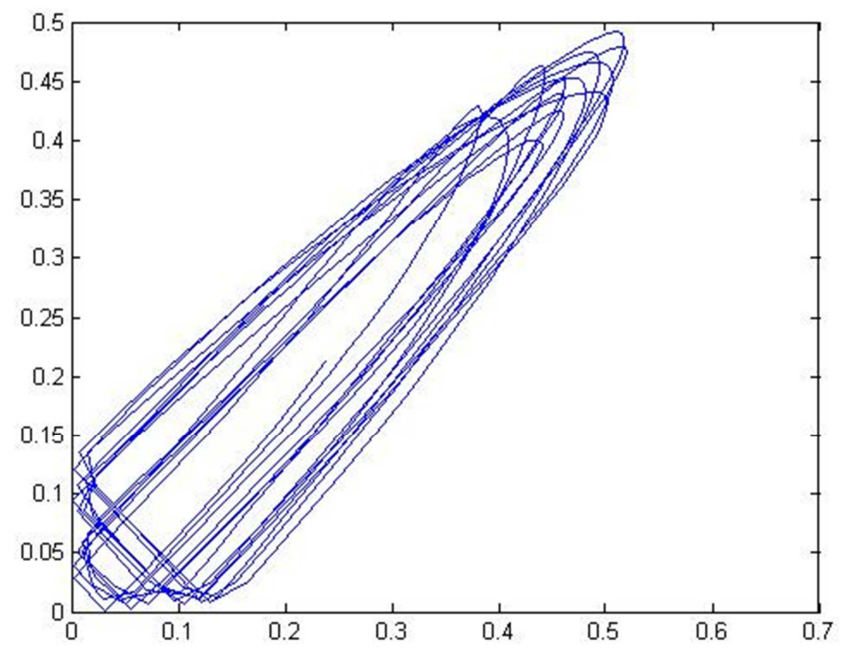

(a)

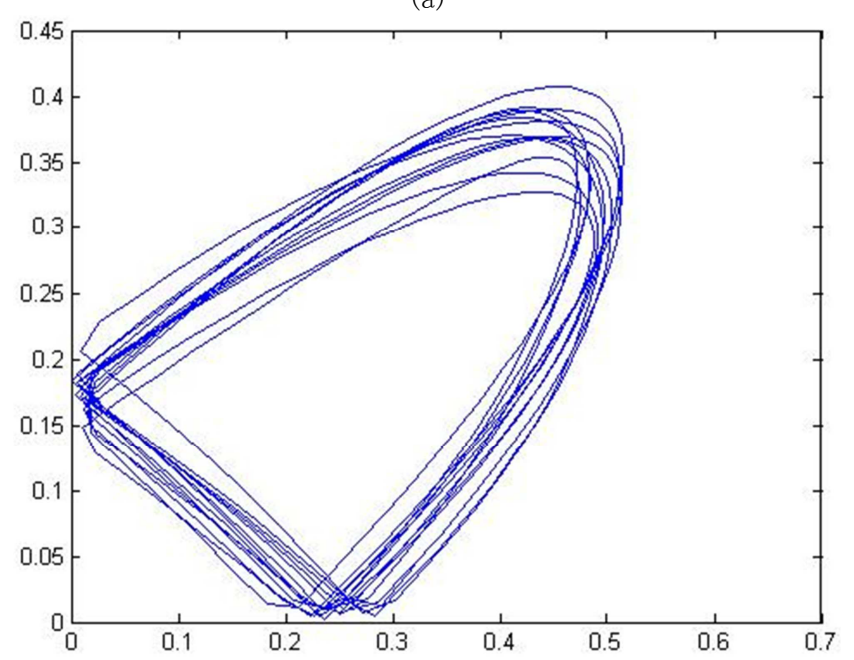

(b)

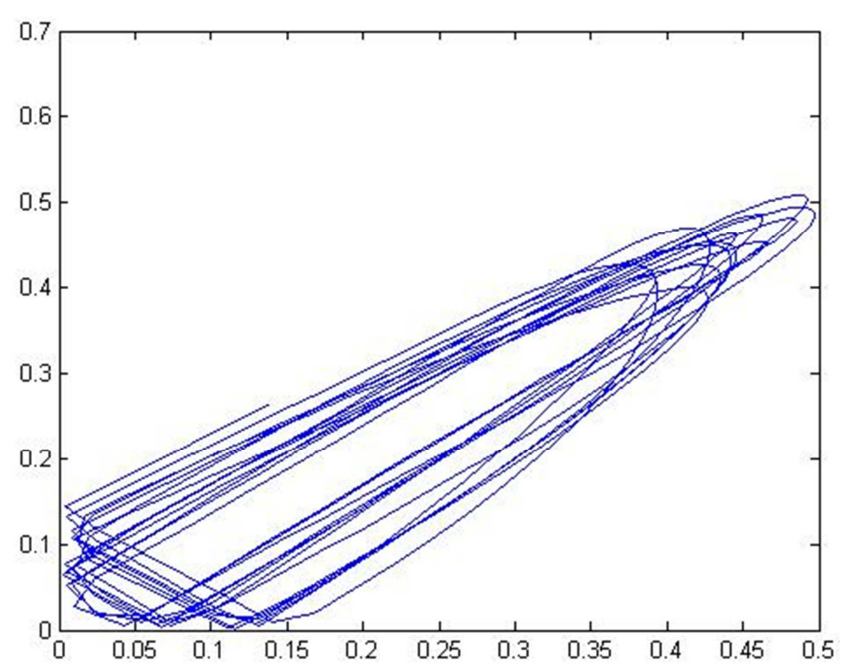

(c)

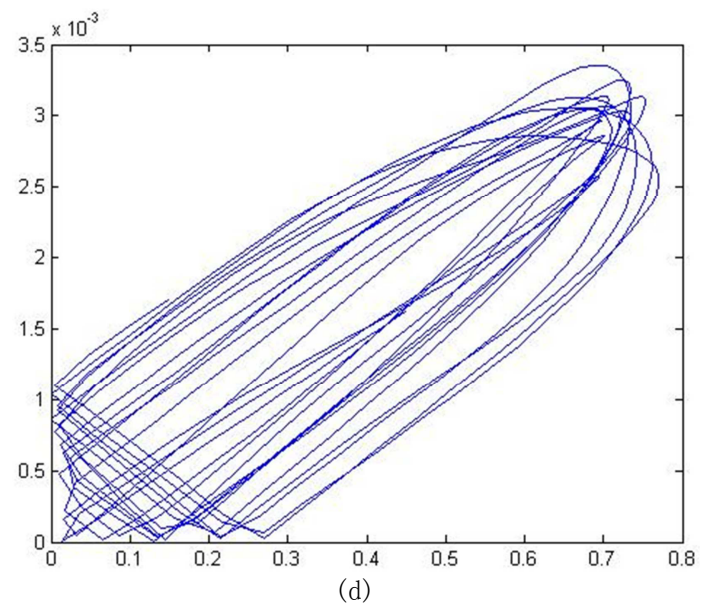

图5 光传输结束时的输出偏振态。

(a) 填充率为 0.25 时二维磁性光子晶体方形空气孔结 构; (b) 磁光晶体结构; (c) 填充率为 0.25 时二维磁性光子 晶体圆形空气孔结构; (d) 无磁性的光子晶体结构

磁性光子晶体结构能够实现光偏振面的改变, 并且很 好的保留其原有的偏振度, 当然, 这只对禁带波长内的光 有效, 对于非禁带的光则不会有此特性, 这也是磁性光子 晶体独有的特性。

影响二维磁性光子晶体偏振的几个因素有: 晶格填充 率, 晶格结构, 磁性角元, 入射光波长等。下面通过控制 变量法来分析这几个因素的影响。

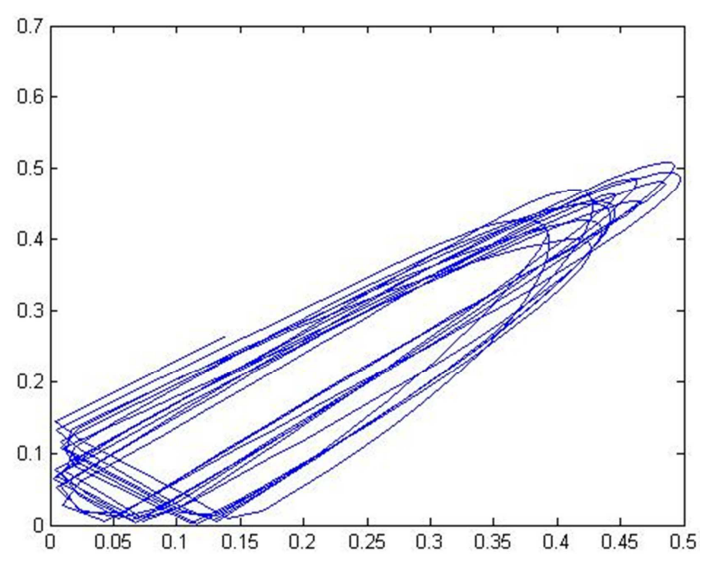

(a)

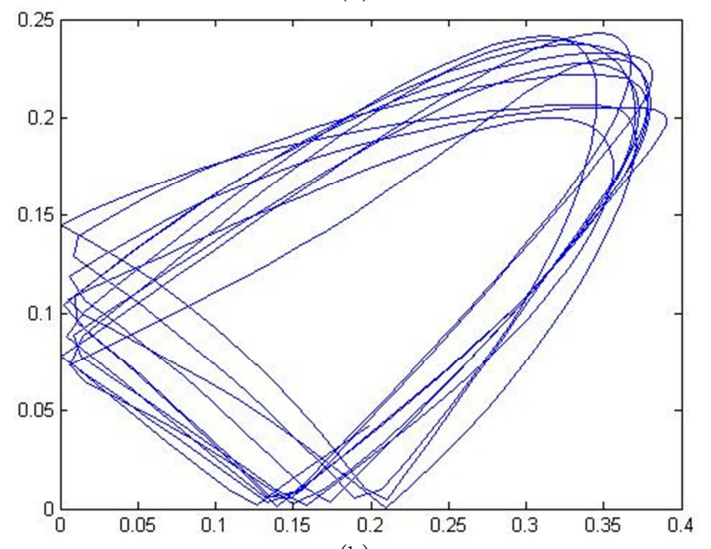

(b) 


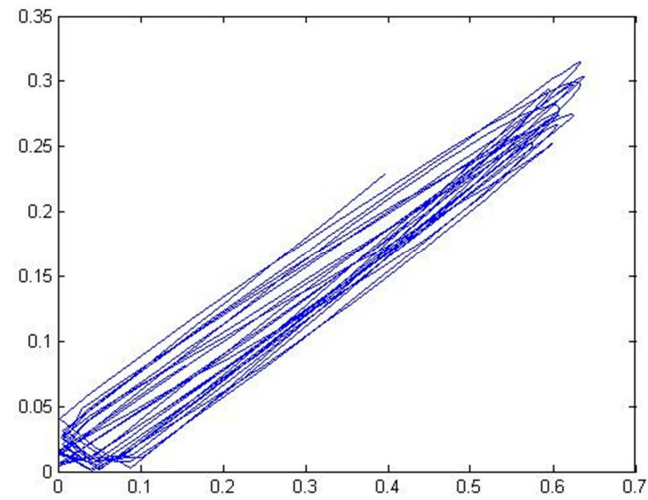

(c)

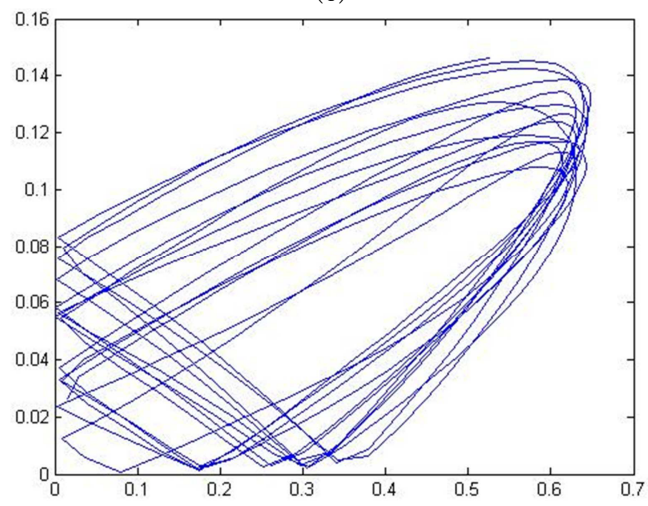

(d)

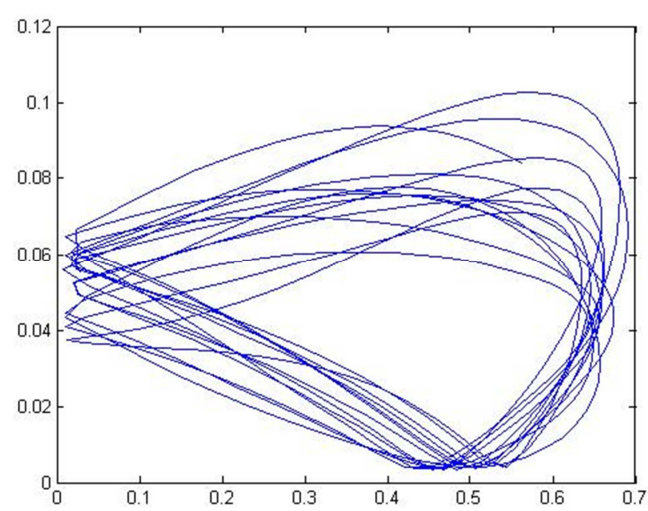

(e)

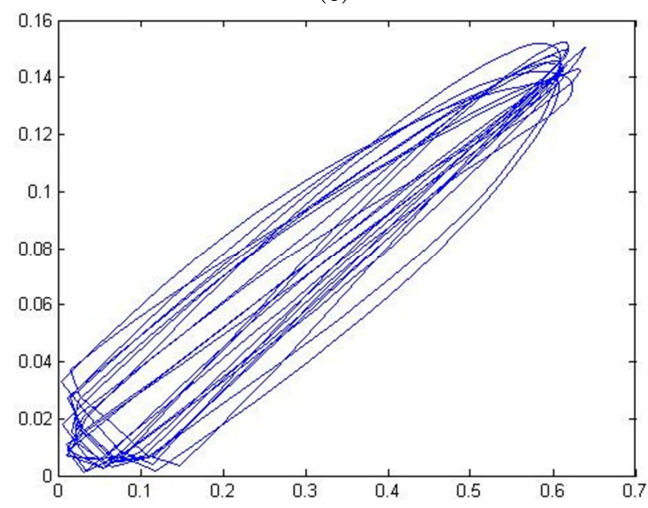

(f)

图6 圆形空气孔模型在不同条件下输出光的偏振态。

(a) 、 (b) 填充率为 0.2 时波长 $1.3 \mathrm{um}$ 和 $1.6 \mathrm{um}$ 结构

(c)、 (d) 波长为 $1.3 \mathrm{um}$ 时填充率为 0.25 和 0.3 结构

(e)、 (f) 填充率 0.3 , 波长为 1.3 um时 $\delta$ 为 $1.5 \mathrm{e}^{-}-1$ 和 $2.5 \mathrm{e}^{-}-1$ 结构
可以看到, 当改变二维磁性光子晶体的晶格结构、入 射光波长、填充率及磁性角元这几个参量时, 可以对输出 光的偏振态进行控制, 主要体现在旋转角度及偏振度上。 磁性角元直接受外界磁场的影响, 因此可以通过改变外部 磁场来控制磁性角元的大小。

\section{3. 结果与讨论}

从上述仿真结果中可以看到, 光在经过磁性光子晶体 结构或是其他磁性介质时, 其偏振态会发生一些变化, 这 一变化会受其结构、入射光波长、磁性角元分量及光子晶 体的填充率的影响而改变其偏振态的椭圆率和旋转角度。 由面内传播的仿真结果表明, 在普通光子晶体结构中禁带 波长内的光波在相同结构的磁性光子晶体中同样处于禁 带 (绝大多数的光会沿着缺陷进行传播), 而在光子晶体 中沿轴向传播时, 有光子晶体结构的材料中输出光有更大 的偏转角和更小的椭圆率, 而相同波长的光, 在磁性角元 及晶格结构相同的情况下, 填充率为 0.25 时的输出偏振态 椭圆率接近为 0 , 增大或减小填充率时椭圆率增大, 不同 的波长传播时, $1.6 \mathrm{um}$ 的光要比 $1.3 \mathrm{um}$ 波长的光椭圆率要大 得多, 同时, 对比不同磁性角元的情况下, 当磁性角元选 取的适当时, 能够得到更大的偏转角和更接近 0 的椭圆率。 因此, 在设置参数为填充率 0.25 , 波长为 $1.3 \mathrm{um}$, 磁性角 元为 $1.98 \mathrm{e}^{-1}$ 的圆形晶格结构中能够得到偏转角为 $45^{\circ}$, 椭 圆率为 0 的线偏振光。并且, 由FDTD算法的仿真结果来看, 光在沿轴向进行传播时, 是螺旋式 [9-11]前进的, 这一结 果有助于从动力学的角度去认识磁光效应的本质。

为了更直观的认识这些偏振态之间的联系, 可以将所 有偏振态全部表征到邦加球上。邦加球的表面可以看作是 表示光偏振态的归一化斯托克斯矢量的所有几何端点的 集合。其表面的任意一点表示了不同的偏振态，方位角表 示在邦加球上的经度，椭圆率（ $\tan \beta=b / a ）$ 表示纬度。 由于本次仿真中入射光偏转角为 $0^{\circ}$, 所以其出射光偏振图 像的偏振角即为其方位角。如下图7所示, S-start为起始 偏振态, $\mathrm{S}-$ end为输出偏振态, 从 $\mathrm{S}-$ start $\mathrm{t}$ 到 $\mathrm{S}-$ end经历了 偏转角和椭圆率的变化。将偏振态引入到邦加球的一大优 点就是可以通过球上坐标直观地看到在不同条件下, 其旋 转角和椭圆率所得到的不同变化, 同时可以帮助我们分析, 如果要得到某种偏振态的输出光应该如何调节磁性光子 晶体的各个参数。此外, 这一定量表征方法也为之后验证 一种新的磁光晶体偏振测量方法提供了思路。

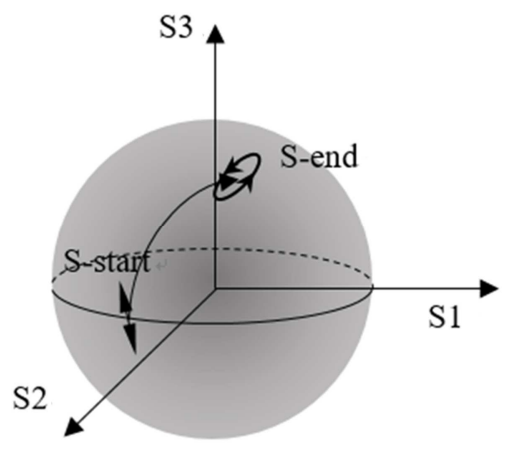

图7 邦加球偏振态变化示意图。 


\section{4. 结论}

以上分析结果表明, 通过改变二维磁性光子晶体的晶 格结构、入射光波长、填充率及外界磁场等参数可以控制 其光子带隙及出射光的偏振态等特性。根据相关文献指出, 光子晶体结构不仅可以改变输出光的椭圆率, 还可以很大 程度增大旋转角, 但在此次仿真中, 两种结构的旋转角相 差并不大, 可能是因为传输距离太短, 磁性角元设置过大 等因素引起的。并且, 本文的不足之处还有对缺陷的引入 没有进行讨论, 这会在之后的工作中加入进去。其次, FDTD 的仿真结果很好的表现出了光在磁性光子晶体中螺旋前 进的特性, 这为从微观层面研究磁光效应本质提供了一定 的理论基础。

\section{致谢}

本文工作为中央高校基本科研业务费资助项目 （HUST-2015049），并部分得到了中国电子集团46所创新 基金支持（CETC N0.46 Research Institute）。

\section{参考文献}

[1] 刘泰康, 赵亚丽等. 光子晶体技术及应用 $[\mathrm{M}]$. 北京: 国防工 业出版社，2015：1-11。

[2] 程敏熙, 何振江, 黄佐华. 偏振光斯托克斯参量的测量和应 用 $[\mathrm{J}]$. 红外与激光工程, 2006, 35 (增刊)：109-115。
[3] 史萌. 光束偏振态斯托克斯参量的实时测量 [D]. 山东: 曲阜 师范大学, 2006: 5-11.

[4] 谢处方, 饶克谨. 电磁场与电磁波 [M]. 北京: 高等教育出版 社, 2006: 70-71。

[5] E. Ate1, D. Veyse1. MATLAB模拟的电磁学时域有限差分法 [M]. 喻志远. 北京：国防工业出版社，2012：2-3。

[6] 张浩, 赵建林, 张晓娟等. 二维磁性光子晶体及其模场分析 [J].物理学报, 2007, 56(06): 3546-3551。

[7] 张浩, 赵建林, 张晓娟. 带缺陷结构二维磁性光子晶体的数 值模拟分析 $[\mathrm{J}]$. 物理学报, 2009, 58 (5)：3532-3537。

[8] V. Dmitriev, G. Portela. Multifunctional two-dimensional photonic crystal optical component based on magneto-optical resonator: nonreciprocal two-way divider-switch, nonreciprocal 120 deg bending-switch, and three-way divider[J]. Optical Engineering, 2014, 53(11): 115102.

[9] Jian Wang, Jeng-Yuan Yang, Irfan M. Fazal, et al. Terabit free-space data transmission employing orbital angular momentum multiplexing[J]. Nature photonics, 2012, 6(7): 488-496.

[10］狄慧鸽, 曾和平, 何志平等. 光束偏振的高精度实时检测技 术 $[J]$. 光学工程, $2012,39(1)$ : 12-16。

[11] 李政勇. 光纤偏振态的高速控制与偏振编码通信 [D]. 北京: 北京交通大学，2009：4-7。 